\title{
Artificial Neural Network for prediction of plasma hormones, liver enzymes and performance in broilers
}

\author{
A. Moharrery ${ }^{1}$ and A. Kargar $^{2}$ \\ ${ }^{1}$ Animal Science Department, Agricultural College, Shahrekord University \\ Shahrekord, Iran \\ ${ }^{2}$ Electrical Department, Engineering College, Shahrekord University \\ Shahrekord, Iran
}

(Received 10 April 2006; revised version 22 January 2007; accepted 2 May 2007)

\begin{abstract}
A three-layer feed forward Artificial Neural Network was trained to predict plasma hormones and liver enzymes in broiler chickens. Six diet parameters are selected as inputs (predictor) and eleven performances, plasma hormones or liver enzymes are chosen as output (predicted) parameters of the model. Birds were fed diets containing different concentration of energy and protein. A data set with 100 individuals is divided in two subsets (each contains 50 individuals) for training and evaluation of the neural network. A comparison is made between laboratory analysis results and the neural network predicted values for plasma hormones and liver enzymes. The neural network accurately forecasted the considered values in the first (training) subset and invalid answers were not identified in the second verification subset. The proposed model were found to most effective identify performance, plasma hormones or liver enzymes for broiler chickens fed the diets on the known range of the energy and protein concentration. Sensitivity analysis shows the dependency evaluation of the output parameters with respect to the inputs.
\end{abstract}

KEY WORDS: liver enzymes, plasma hormones, neural network, body weight, feed conversion

\section{INTRODUCTION}

An Artificial Neural Network (ANN) is an information-processing paradigm that is inspired by the way of biological nervous systems, such as the way the brain processed information. ANNs, like people, learn by example. An ANN will

\footnotetext{
${ }^{1}$ Corresponding author: e-mail: alimoh@mailcity.com
} 
be configured for a specific application, such as pattern recognition, prediction of financial indices, optimization of chemical processes, identification of cancerous cells, recognition of chromosomal abnormalities, detection of ventricular fibrillation (Cheng and Titterington, 1994; Buchmann et al., 2002) or data classification through a learning process. Learning in biological systems involves adjustments to the synaptic connections that exist between the neurons.

ANNs learn from patterns of interactions, without requiring a priori knowledge of relations between the variables under investigation. An ANN is a set of processing units (or neurons) that are interconnected by a set of weights (analogous to synaptic connections in the nervous system) which allow both serial and parallel processing of the information through the network (Astion and Wilding, 1992; Roush et al., 1996). The neurons may receive excitatory or inhibitory inputs from other neurons (Forsstrm and Dalton, 1995), and produce an output that is usually a non-linear function of the inputs (Astion and Wilding, 1992). Regarding its use in poultry science, Roush et al. (1996) studied the prediction of ascites in broilers using ANNs, comparing diagnostics results with the incidence predicted by the ANN.

It has been reported that dietary fat levels can influence lipid metabolism in birds. Balnave and Pierce (1969) reported that dietary fat decreased the in vitro incorporation of acetate into hepatic fatty acids. Goodridge et al. $(1989,1996)$ demonstrated that nutritionally and hormonally induced changes in hepatic enzyme activity were accompanied by comparable changes in enzyme synthesis. Certain studies in the past have examined dietary energy influences on plasma thyroid hormones. For example, Schalch and Cree (1985) found that reducing fat calories decrease body weight, $\mathrm{T}_{3}$ and $\mathrm{T}_{4}$ in young rats. A later report from this group (Yang et al., 1987) indicated that reducing carbohydrate energy also decrease body weight, but did not change either $\mathrm{T}_{3}$ or $\mathrm{T}_{4}$.

This paper aims to study the use of ANNs to estimate performance - outputs (body weight, feed conversion, enzyme pattern in the broiler liver, some plasma hormones, etc.), based on specified variables - inputs (metabolizable energy, fat, carbohydrate, crude protein, metabolizable carbohydrate and organic matter) in broiler chickens. In addition, sensitivity analysis has been used to show the dependency of the output parameters with respect to the inputs.

\section{MATERIAL AND METHODS}

\section{Data}

The data in relation to the plasma hormones [insulin-like growth factor-I (IGF-I), growth hormone $(\mathrm{GH})$, triiodothyronine $\left(\mathrm{T}_{3}\right)$ and thyroxine $\left(\mathrm{T}_{4}\right)$ ] and in vitro lipogenesis (IVL), has been taken from the male, Indian River broiler chicken which 
were assigned to the one of the eight dietary treatments (Rosebrough et al., 1999). The results were diets containing either two levels of $\mathrm{CP}(120$ or $190 \mathrm{~g} / \mathrm{kg})$ and either 300 , 600 or $1200 \mathrm{kcal} \mathrm{ME}$ from fat. Two additional experimental diets contained either 234 or $285 \mathrm{~g} \mathrm{CP} / \mathrm{kg}$ and $300 \mathrm{kcal} \mathrm{ME}$ from fat. The energy contributions of fat and $\mathrm{CP}$ were calculated as 9.0 and $4.0 \mathrm{ME} / \mathrm{g}$, respectively. The remainder of the energy in each diet was assumed to be carbohydrate and was calculated by subtraction.

The data in relation to the body weight (BW), feed intake (FI), feed efficiency (FE) and liver enzymes including malate, nicotinamide adenine dinucleotide phosphate oxidoreductase-[decarboxylating] (MDH-NADP) and isocitrate, nicotinamide adenine dinucleotide phosphate oxidoreductase [decarboxylating] (IDH-NADP), has been taken from broiler chicken (Ross) (Rosebrough et al., 1999; Moharrery, 2005).

\section{Neural Networks structure}

Selecting inputs and outputs, number of layers, and number of neurons in each layer of the ANNs can affect benefits and abilities of them, significantly. In present work, parameters: metabolizable energy [ME $(\mathrm{Kcal} / \mathrm{Kg})]$, fat $(\mathrm{g})$, carbohydrate $[\mathrm{CHO}(\mathrm{g})]$, crude protein $[\mathrm{CP}(\mathrm{g})]$, metabolizable carbohydrate $[\mathrm{MCHO}(\mathrm{g})]$, and organic matter $[\mathrm{OM}(\mathrm{g})]$ are selected as the inputs (predictors) of the model and also quantities: body weight (BW), feed intake (FI), feed efficiency (FE), in vitro lipogenesis (IVL), MDH-NADP, ICD-NADP, aspartate aminotransferase (AAT), insulin-like growth factor-I (IGF-I), growth hormone $(\mathrm{GH})$, triiodothyronine $\left(\mathrm{T}_{3}\right)$ and thyroxine $\left(\mathrm{T}_{4}\right)$ are considered as the outputs (predicted values). Thus, we have a system with six inputs and eleven outputs.

For modeling of each mentioned eleven output quantities (a six input and one output), 3-layer feed forward ANN is chosen. There are three neurons in the input layer, two neurons in the hidden layer, and one neuron in the output layer as shown in Figure 1. Solid circles in this figure show input and output nodes and other circles show neurons of the ANN. The number of the layers in the

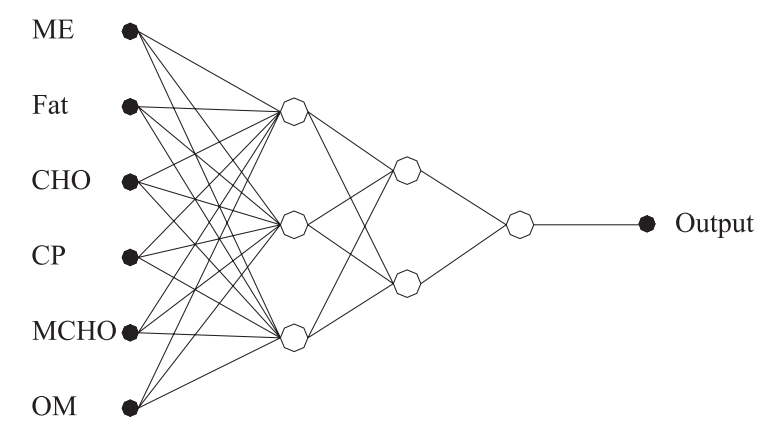

Figure 1. The proposed ANNs structure 
ANNs and the number of the neurons in each layer are designated by the trial and error method. This structure for ANNs is selected from more than 400 different structures (with different layers and different neurons in each layer) on basis of the mean square of the error (MSE) measure. For normalizing inputs and outputs of the ANNs, all values in each input/output node are divided to the maximum value in that node. Thus, all input/output values in the network will be kept in the range of ( 0 to 1$)$. The hyperbolic tangent transfer function is used for all neurons in the ANNs. This transfer function is shown in Figure 2, which gives an appropriate response for many applications with respect to linear transfer function. The linear transfer function is presented in the figure by dash lines as well. In order to simulation of the ANNs, the MATLAB software (MathWorks, 2004) was used.

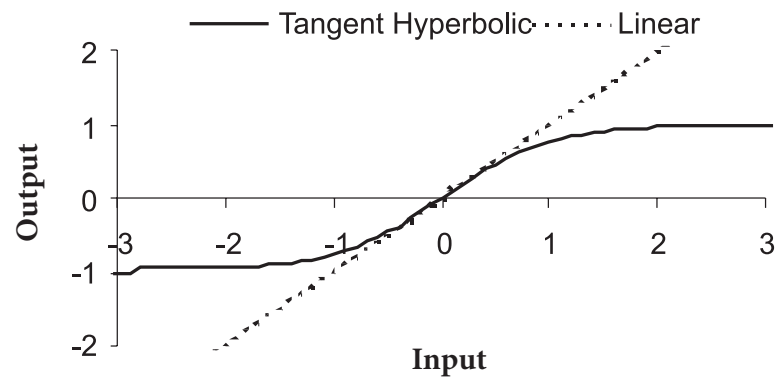

Figure 2. The ANNs neurons transfer function

Training of the ANNS

Selecting a suitable data set for training of the ANNs is very significant. If a small number of data (consultable and incomplete set of data) selects as training data for the ANNs, the ability of the network encounter with an unknown (not used in the training set) record will be reduced (Rivals and Personnaz, 2003). In present work, a data set with 100 individuals (records) is divided randomly in two subsets (each contains 50 individuals) for training and evaluation of the ANNs. The mean along with maximum, minimum and standard deviation values of the data set are shown in the Table 1. Training is accomplished after

Table 1. Mean, maximum, minimum, and standard deviation (SD) of data set

\begin{tabular}{lcccc}
\hline Item & Mean & Max & Min & SD \\
\hline ME, Kcal. $\mathrm{Kg}^{-1}$ & 3322 & 3470 & 3193 & 120.1 \\
Fat, g & 121.5 & 252.5 & 52.77 & 54.52 \\
CHO, g & 1197 & 1316 & 1088 & 60.28 \\
CP, g & 324.8 & 511 & 196.3 & 110.8 \\
MCHO, g & 881.6 & 1109 & 643.5 & 109 \\
OM, g & 1644 & 1836 & 1464 & 128 \\
\hline
\end{tabular}

ME: metabolizable energy in the diet; $\mathrm{CHO}$ : carbohydrate, $\mathrm{CP}$ : crude protein; MCHO: metabolizable carbohydrate; OM: organic matter; ${ }^{1}$ gram intake during growing period 
normalizing (input and output) of the selected samples in 500 epochs (training stage) with BPE (Back Propagation of Error) procedure.

\section{Sensitivity analysis}

In order to evaluate the proposed ANN based model, a sensitivity analysis is accomplished as follows. Three important inputs (fat, carbohydrate and crude protein) are chosen and sensitivities of all eleven outputs with respect to these inputs are analysed. Due to the dependency of the other inputs (metabolizable energy, metabolizable carbohydrate, and organic matter) to the important inputs, only in the sensitivity analysis, those inputs are not considered and discarded. Thus, the inputs of the models in the sensitivity analysis will be reduced to three.

Assume that $I n \_\max (j)$ and $I n \_\min (j)$ are maximum and minimum of the $j$-th input, respectively. Thus, the difference of the $\mathrm{j}$-th input (for $\mathrm{j}=1,2,3$ ) between these values is:

$$
I n \_v a r(j)=I n \_\max (j)-I n \_\min (j)
$$

Also, assume that $O u t_{-} \max (k, j)$ and $\operatorname{Out} \min (k, j)$ are the k-th outputs corresponding to the $I n \_\max (j)$ and $I n \_\min (j)$ (while other inputs are held constant at their average values, and $\mathrm{k}=1, \ldots, 11)$. We define the sensitivity of the $\mathrm{k}$-th output with respect to the $\mathrm{j}$-th input as follows:

$$
S(k, j)=\frac{O u t_{-} \max (k, j)-O u t_{-} \min (k, j)}{I n_{-} \max (j)-I_{-} \min (j)}
$$

Criteria of goodness of the approach

For comparison between observed and predicted values a suitable method, which explained by Zhao et al. (2001) is used. In this method, bias and accuracy factors are used as a quantitative measure of the goodness of the prediction model. The bias factor indicates by how much, on average, a model over-predicts (bias factor $>1$ ) or under-predicts (bias factor $<1$ ) the observed data.

$$
\text { Bias factor }=10^{\frac{1}{n} \sum_{i=1}^{n} \log _{10}\left(\frac{\text { predicted value }_{i}}{\text { observed value }_{i}}\right)}
$$

The accuracy factor indicates by how much, the predictions differ from the observed data.

$$
\text { Accuracy factor }=10^{\frac{1}{n} \sum_{i=1}^{n}} \log _{10}\left(\frac{\text { predicted value }}{\text { observed value }}\right) \mid
$$

In both equations, $n$ is the number of observations used in the calculations. In a perfect model, both the bias and accuracy factors are equal to one. 
Additionally, we used t-Student to compare the predicted values with the results of laboratory assays using SigmaStat software (SigmaStat, 1994).

\section{RESULTS AND DISCUSSION}

Prediction accuracy of the neural networks is highly dependent on the quality of the training data. For evaluating of the proposed model, the complete set of data (including new data and training data) is used. The errors (difference between predicted and observed values) for all output characters are shown in Figures 313. In each figure, the error has been shown in relation to the main three parts of the diet composition. Note that, other three parts of the diet composition only discarded in the presentations and kept in the inputs of the ANN models. In most characters, carbohydrate consumption is the main factor for the error, because carbohydrate is the main part of each diet. ANNs can often describe complex relationships between input and output variables that are not readily apparent when inspecting a database or using other prediction methods (Baxt, 1995). These predictions were compared with chemically analysed composition values in the database, found to be highly accurate. More accurate predictions of feed intake, feed efficiency, and chicken growth, may also address widespread nutrient management concerns (in part) by reducing excess nitrogen resulting from oversupplementation of protein.

As shown in Figures 3-13 the high sensitivity and specificity obtained with the complete data set support the hypothesis that minimally invasive measurements may provide enough information to successfully predict hormonal status or enzyme pattern in the broiler in response to different energy or protein in the diets.

The means of observed and predicted values along with bias and accuracy factors are reported in Table 2. To compare the predicted values with the results of laboratory assays, t-Student was used. No significant difference was detected between original data and predicted values for all parameters.
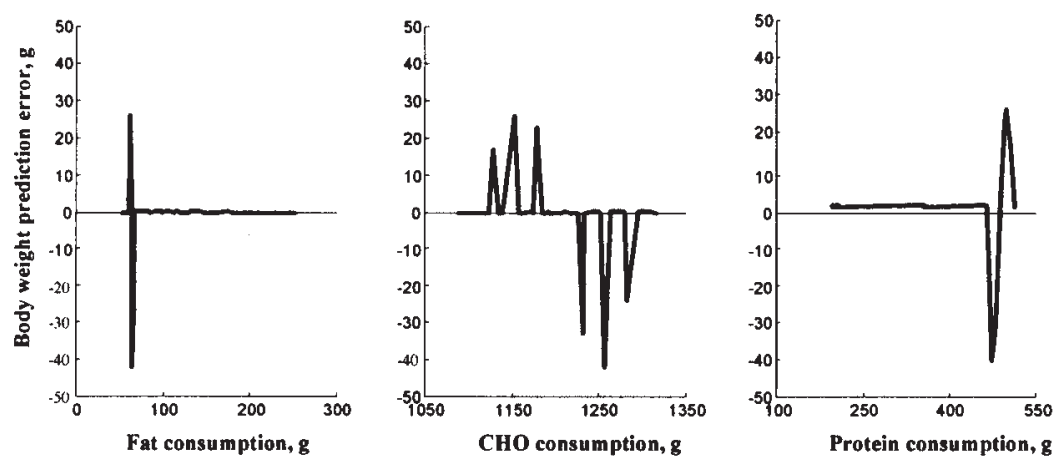

Figure 3. Difference between predicted and observed values for weight of chicken in $28 \mathrm{~d}$ of age 

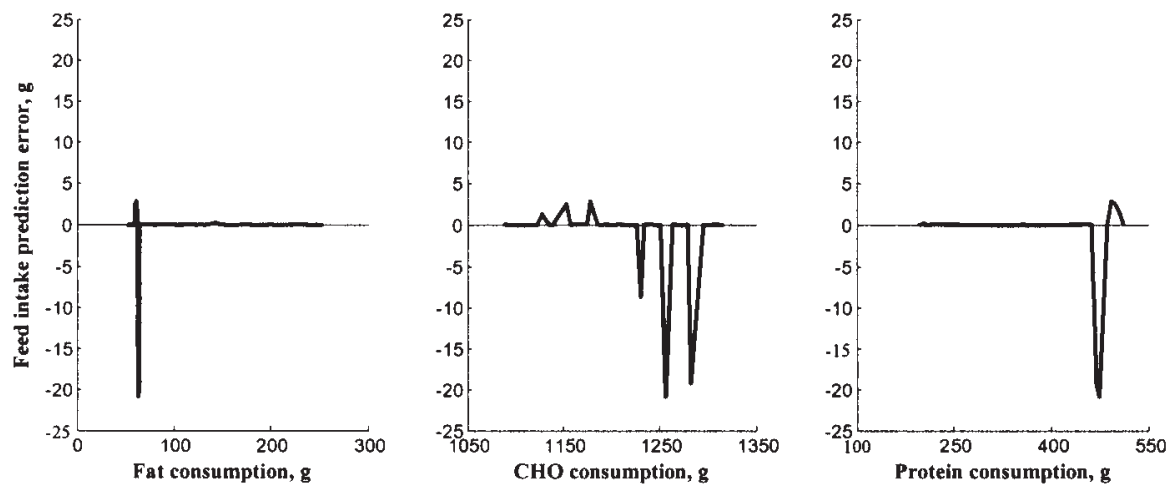

Figure 4. Difference between predicted and observed values for feed intake on chicken from 7 to 28 d of age
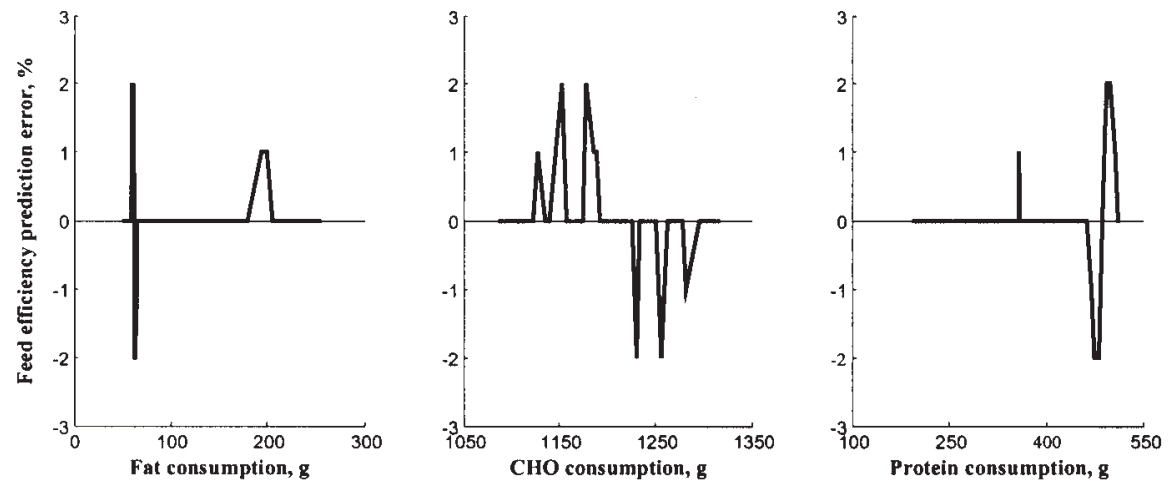

Figure 5. Difference between predicted and observed values for feed efficiency on chicken from 7 to $28 \mathrm{~d}$ of age
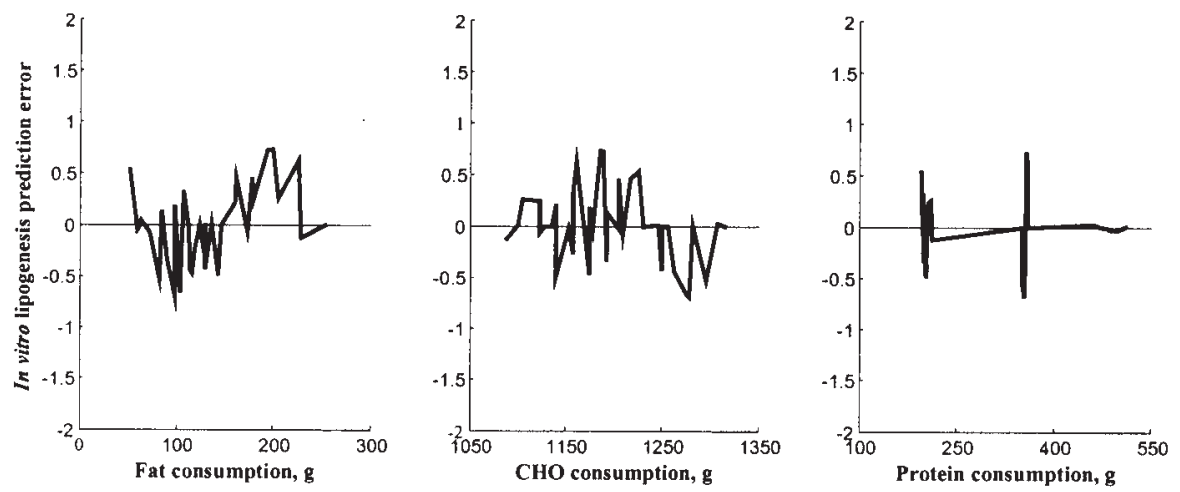

Figure 6. Difference between predicted and observed values for in vitro lipogenesis in broiler chicken in $28 \mathrm{~d}$ of age. Values are expressed as micromoles substrate incorporated per gram of liver 

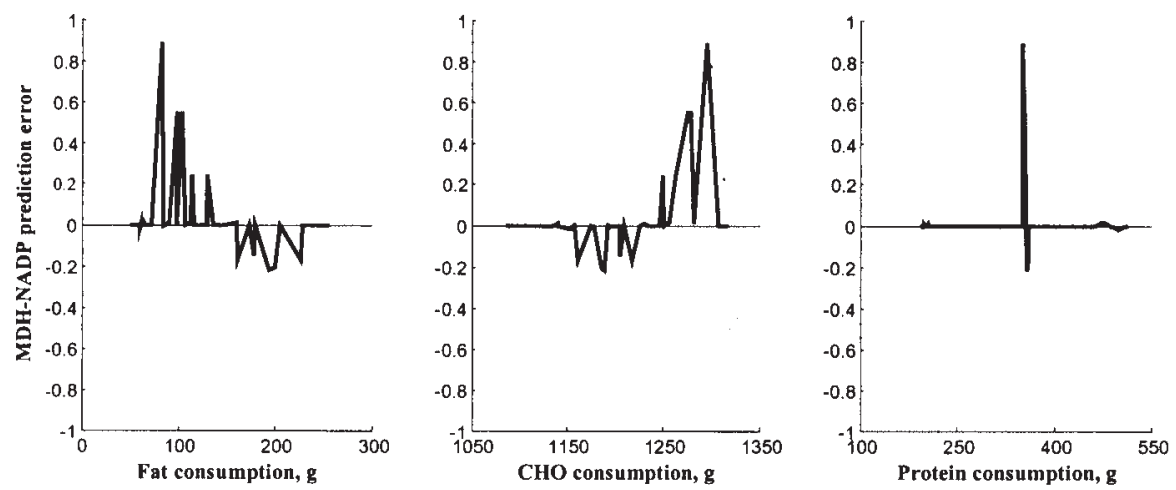

Figure 7. Difference between predicted and observed values for malate-NADP oxidoreductase (MDH-NADP) in broiler chicken in $28 \mathrm{~d}$ of age. Enzyme activity is noted as micromoles of oxidized or reduced $\mathrm{NAD}(\mathrm{P})$ per minute per gram of liver at $30^{\circ} \mathrm{C}$
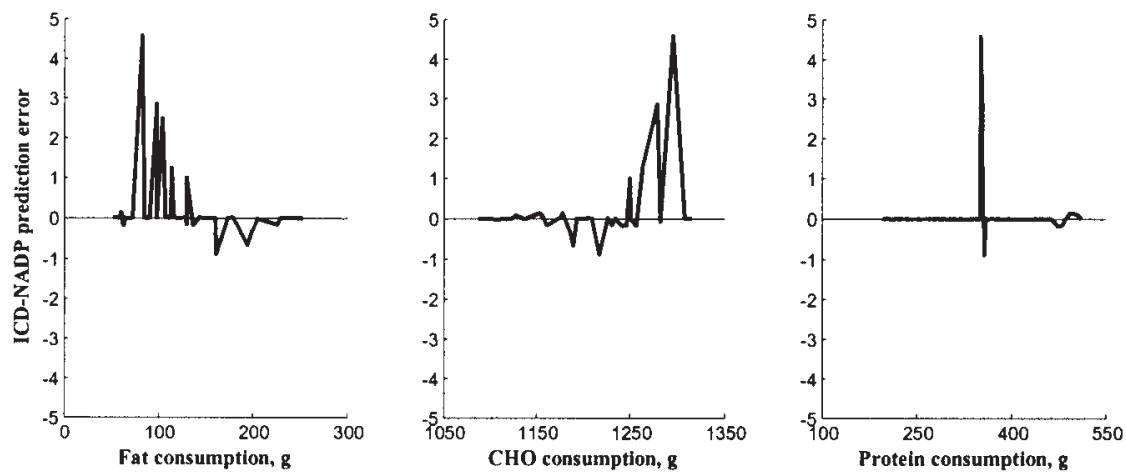

Figure 8. Difference between predicted and observed values for isocitrate-NADP oxidoreductase (ICD-NADP) in broiler chicken in $28 \mathrm{~d}$ of age. Enzyme activity is noted as micromoles of oxidized or reduced $\mathrm{NAD}(\mathrm{P})$ per minute per gram of liver at $30^{\circ} \mathrm{C}$
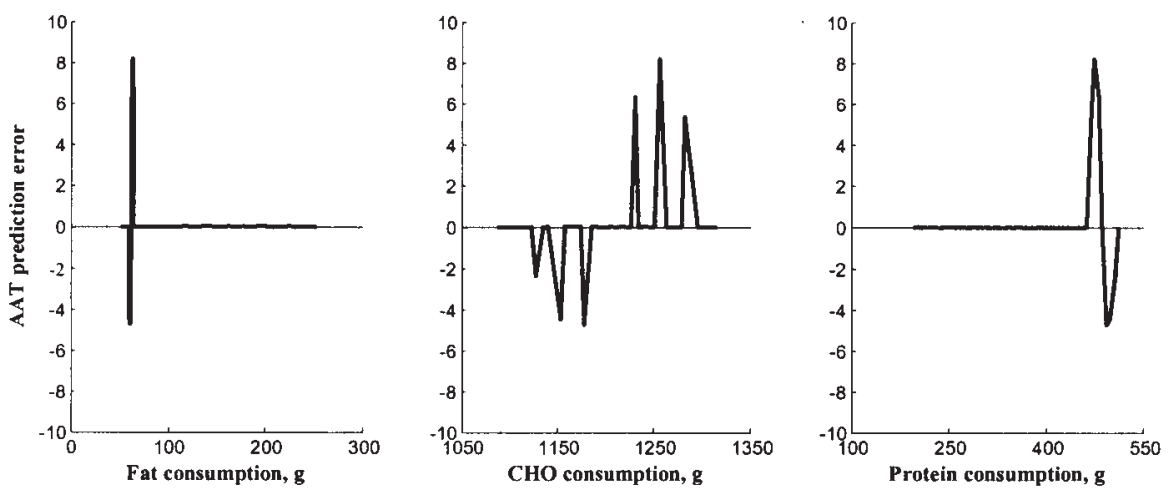

Figure 9. Difference between predicted and observed values for aspartate aminotransferase in broiler chicken in $28 \mathrm{~d}$ of age. Enzyme activity is noted as micromoles of oxidized or reduced NAD(P) per minute per gram of liver at $30^{\circ} \mathrm{C}$ 

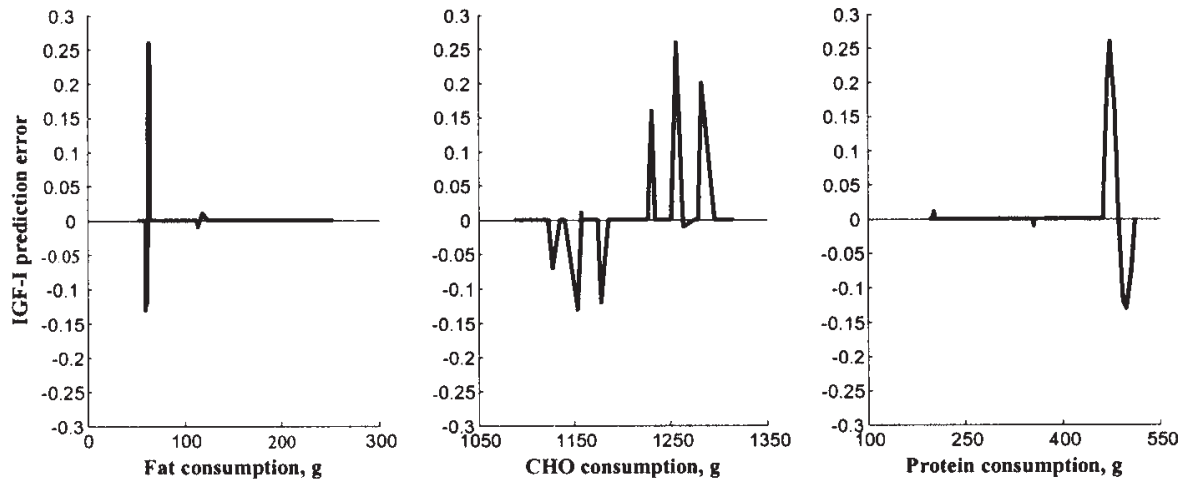

Figure 10. Difference between predicted and observed values for insulin-like growth factor-I (IGF-I) in broiler chicken in $28 \mathrm{~d}$ of age. The hormone concentration is reported as $10^{-9} \mathrm{M}$
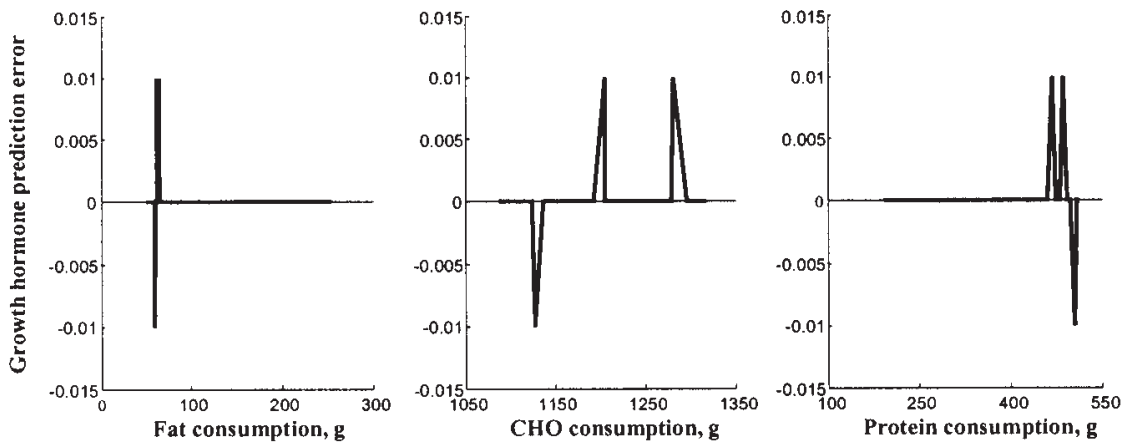

Figure 11. Difference between predicted and observed values for growth hormone in broiler chicken in $28 \mathrm{~d}$ of age. The hormone concentration is reported as $10^{-9} \mathrm{M}$
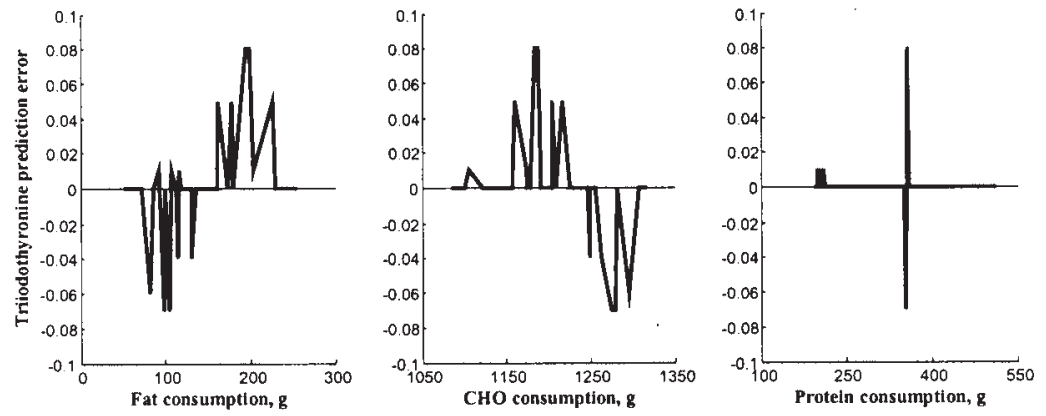

Figure 12. Difference between predicted and observed values for triiodothyronine (T3) in broiler chicken in $28 \mathrm{~d}$ of age. The hormone concentration is reported as $10^{-9} \mathrm{M}$ 

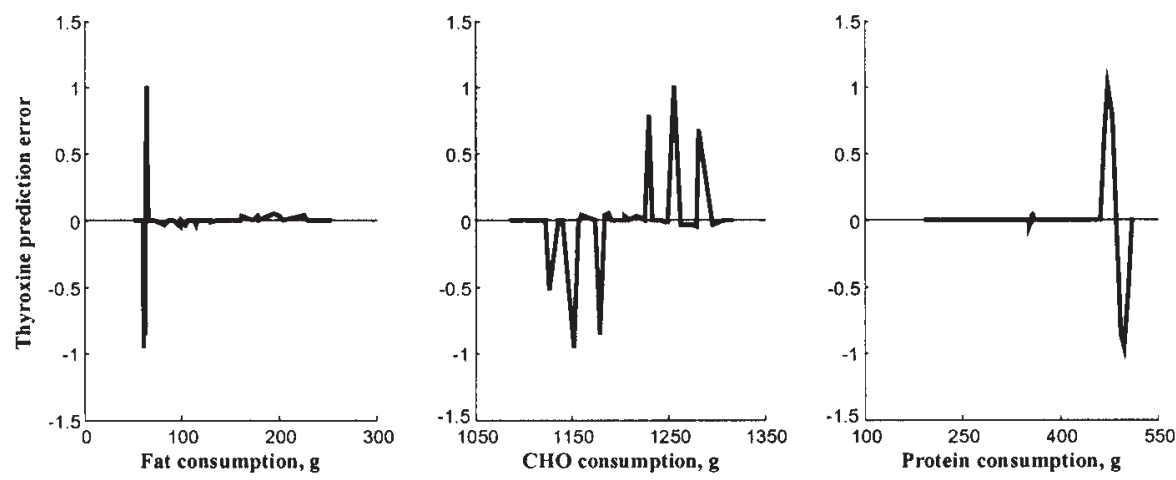

Figure 13. Difference between predicted and observed values for thyroxine (T4) in broiler chicken in $28 \mathrm{~d}$ of age. The hormone concentration is reported as $10^{-9} \mathrm{M}$

Additionally, we used bias and accuracy factors to compare the goodness of estimations. Bias and accuracy factors indicate a close degree of goodness of fit between observed and ANNs predicted values. The discrepancy between bias and accuracy factors is negligible for each character. However, for the reason that the observed value is in the denominator of both equations for bias and accuracy factors, and we do not have any zero in the observed or predicted values, using this method is logical for system evaluation. One of the most important advantages of the ANN models is that they predict values accurately, because no values were closer to zero or one. This finding is disagreement with Zhao et al. (2001), that they mentioned due to the limitation of their formulae, bias and accuracy factors

Table 2. Comparison of results for observed and ANNs predicted values

\begin{tabular}{llllllllllll}
\hline Parameter & BW & FI & FE & IVL & MDH & ICD & AAT & IGF & GH & T $_{3}$ & $T_{4}$ \\
\hline Mean & & & & & & & & & & & \\
Observed & 1009 & 1782 & 49.1 & 28.36 & 15.17 & 25.52 & 49.33 & 3.318 & 1.046 & 4.302 & 13.08 \\
Predicted $^{1010}$ & 1783 & 49.1 & 28.37 & 15.13 & 25.29 & 49.13 & 3.311 & 1.046 & 4.301 & 13.08 \\
P value $^{1}$ & 0.9972 & 0.9587 & 0.9382 & 0.9133 & 0.9719 & 0.9238 & 0.9161 & 0.8845 & 0.9969 & 0.9945 & 0.7926 \\
${\text { Error, } \%^{2}}^{0}$ & 0.084 & 0.058 & -0.017 & 0.017 & -0.248 & -0.911 & -0.403 & -0.218 & 0.019 & -0.022 & -0.033 \\
SD & 1.095 & 0.261 & 1.450 & 1.220 & 1.328 & 3.777 & 4.334 & 2.082 & 0.284 & 0.711 & 2.425 \\
Bias & 1.0005 & 1.0005 & 0.9996 & 0.9989 & 0.9979 & 0.9880 & 0.9946 & 0.9981 & 1.0000 & 0.9998 & 0.9993 \\
Accuracy & 1.0032 & 1.0007 & 1.0075 & 1.0089 & 1.0057 & 1.0187 & 1.0146 & 1.0062 & 1.0023 & 1.0037 & 1.0071 \\
MSE & 0.947 & 4.705 & 0.698 & 0.344 & 0.203 & 0.980 & 2.124 & 0.064 & 0.003 & 0.030 & 0.314 \\
\hline
\end{tabular}

BW: body weight; FI: feed intake; FE: feed efficiency; IVL: in vitro lipogenesis; MDH: malate dehydrogenase; ICD: isocitrate dehydrogenase; AAT: aspartate aminotransferase; IGF: insulin-like growth factor-I; $\mathrm{GH}$ : growth hormone; $\mathrm{T}_{3}$ : triiodothyronine; $\mathrm{T}_{4}$ : thyroxine

${ }^{1}$ this value show a statistically significant difference between observed and predicted values

${ }^{2}$ error percentage $=((($ predicted - observed $) /$ observed $) * 100)$

SD: standard deviation of the mean (error)

MSE: mean square of the error 
cannot use for evaluation data which is equal or close to zero or one. As a result, bias and accuracy factors show that prediction values by ANN models are closely to observed values. In this regard, bias and accuracy factors demonstrate no overprediction or under-prediction for data set. In respect to the data set (without zero value), if the bias factor is one, a conclusion made for model goodness.

The weights of relative importance of the dietary variables analysed in the study are shown in Table 3. Percentage of the contribution of each input factor

Table 3. Dietary factors and their neural network contribution

\begin{tabular}{lcccrrrrrrrr}
\hline Dietary & \multicolumn{10}{c}{ Contribution to neural network, \% } \\
\cline { 2 - 12 } factor $^{1}$ & BW & FI & \multicolumn{1}{c}{ FE } & \multicolumn{1}{c}{ IVL } & MDH & ICD & AAT & IGF & GH & \multicolumn{1}{c}{$\mathrm{T}_{3}$} & \multicolumn{1}{c}{$\mathrm{T}_{4}$} \\
\hline Fat & 10.66 & 13.31 & 12.91 & 9.37 & 9.17 & 10.67 & 14.84 & 17.72 & 7.63 & 11.00 & 8.98 \\
CHO & 64.13 & 61.79 & 64.13 & 84.62 & 78.24 & 73.22 & 70.38 & 50.62 & 64.83 & 57.92 & 46.51 \\
CP & 25.21 & 24.90 & 22.96 & 6.02 & 12.59 & 16.11 & 14.78 & 31.67 & 27.54 & 31.09 & 44.51 \\
\hline
\end{tabular}

BW: body weight; FI: feed intake; FE: feed efficiency; IVL: in vitro lipogenesis; MDH: malate dehydrogenase; ICD: isocitrate dehydrogenase; AAT: aspartate aminotransferase; IGF: insulin-like growth factor-I; $\mathrm{GH}$ : growth hormone; $\mathrm{T}_{3}$ : triiodothyronine; $\mathrm{T}_{4}$ : thyroxine

${ }^{1}$ fat, $\mathrm{CHO}$ (carbohydrate), and $\mathrm{CP}$ (crude protein) as a gram intake during growing period

was made with the ANNs. The contribution was based on the sum of the absolute values of the weights leading from the particular variable. The contributions of inputs of the ANNs were analysed to identify important dietary factors affecting performance and physiological parameters of the broiler chicken. The top factor is the $\mathrm{CHO}$ consumption during the growing period. The importance of the $\mathrm{CHO}$ consumption confirms the major contribution of this dietary component to the performance and other physiological parameters to compare other dietary component. The second rank is belonged to the crude protein and the last for the fat.

\section{Practical implications}

The current study proposes that nutritional measurements in the diets may be used as inputs in an ANN to predict the physiological parameters of individual broiler chickens. Individuals identified in this fashion then can be used for selection of best lines or in nutritional or physiological studies. In addition, the method allows decision-making by the technical staff for different production flocks to be based on scientifically obtained objective criteria. Furthermore, this network allows the simulation of consequences following these decisions, also providing the contribution percentage of each dietary component to the poultry production variables under study.

In addition, this method allows the simulation of enzyme pattern in the liver, also providing the contribution of plasma hormones from each type of diets to the broiler physiology variables under study. In this regard, the ANN approach seemed quite satisfactory and the performance obtained was very high (around $99 \%$ ), it seems to be acceptable by the broiler researchers. 


\section{ACKNOWLEDGMENTS}

We are appreciating from Professor B. Rosebrough for his encouragements. We are grateful to all the staff from the Section of Research Department in the Shahrekord University.

\section{REFERENCES}

Astion M.L., Wilding P., 1992. The application of backpropagation neural networks to problems in pathology and laboratory medicine. Arch. Pathol. Lab. Med. 116, 995-1001

Balnave D., Pierce J., 1969. Adaptation of laying hens (Gallus domesticus) to dietary fat with special reference to changes in liver and ovarian lipid content and liver enzyme activity. Comp. Biochem. Physiol. 29, 539-550

Baxt W.A.G., 1995. Application of artificial neural networks to clinical medicine. Lancet 346, 1135 1138

Buchmann N.B., Josefsson H., Cowe I.A., 2002. Performance of European Artificial Neural Network (ANN) calibrations for moisture and protein in cereals using the Danish Near Infrared Transmission (NIT) network. In Focus 26, 5-5

Cheng B., Titterington D.M., 1994. Neural Networks: a review from a statistical perspective. Stat. Sci. 9, 2-54

Forsstrm J.J., Dalton K.J., 1995. Artificial neural networks for decision support in clinical medicine. Ann. Med. 27, 509-517

Goodridge A.G., Crish J.F., Hillgartner F.B., Wilson S.B., 1989. Nutritional and hormonal regulation of the gene for avian malic enzyme. J. Nutr. 119, 299-308

Goodridge A.G., Klautky S.A., Fantozzi D.A., Baillie R.A., Hodnett D.W., Chen W., Thurmond D.C., Xu G., Roncero C., 1996. Nutritional and hormonal regulation of expression of the gene for malic enzyme. Prog. Nucl. Acid Res. Mol. Biol. 52, 89-121

MathWorks, 2004. MATLAB Neural Networks Toolbox. Version 7.0.0.19920 (R14)

Moharrery A., 2005. Effect of malic acid on some serum metabolite and hepatic enzymes in chickens. Int. J. Poultry Sci. 4, 436-441

Rivals I., Personnaz L., 2003. Neural-network construction and selection in nonlinear modeling. IEEE trans. Neural Networks 14, 804-819

Rosebrough R.W., McMurtry J.P., Vasilatos-Younken R., 1999. Dietary fat and protein interactions in the broiler. Poultry Sci. 78, 992-998

Roush W.B., Kirby Y.K., Cravener T.L., Wideman Jr. R.F., 1996. Artificial neural network prediction of ascites in broilers. Poultry Sci. 75, 1479-1487

Schalch D.S., Cree T.C., 1985. Protein utilization in growth: Effect of lysine deficiency on serum growth hormone, somatomedins, insulin, total thyroxine and triiodothyronine, free T4 index, and total corticosterone. Endocrinology 117, 667-673

SigmaStat, 1994. SigmaStat vesion 1.0, Statistical Software, Jandel Corporation

Yang H., Cree T.C., Schalch D.S., 1987. Effect of a carbohydrate-restricted, calorie-restricted diet on the growth of young rats and on serum growth hormone, somatomedins, total thyroxine and triiodothyronine, free T4 index and corticosterone. Metabolism 26, 794-798

Zhao L., Chen Y., Schaffner D.W., 2001. Comparison of logistic regression and linear regression in modeling percentage data. Appl. Environ. Microbiol. 67, 2129-2135 